\title{
On the theory of relaxation in nonlinear elasticity with constraints on the determinant
}

\author{
October 10, 2018 \\ Sergio Conti ${ }^{1}$ and Georg Dolzmann ${ }^{2}$ \\ ${ }^{1}$ Institut für Angewandte Mathematik, Universität Bonn \\ 53115 Bonn, Germany \\ ${ }^{2}$ Fakultät für Mathematik, Universität Regensburg, \\ 93040 Regensburg, Germany
}

\begin{abstract}
We consider vectorial variational problems in nonlinear elasticity of the form $I[u]=\int W(D u) d x$, where $W$ is continuous on matrices with positive determinant and diverges to infinity along sequences of matrices whose determinant is positive and tends to zero. We show that, under suitable growth assumptions, the functional $\int W^{\mathrm{qc}}(D u) d x$ is an upper bound on the relaxation of $I$, and coincides with the relaxation if the quasiconvex envelope $W^{\text {qc }}$ of $W$ is polyconvex and has $p$-growth from below with $p \geq n$. This includes several physically relevant examples. We also show how a constraint of incompressibility can be incorporated in our results.
\end{abstract}

\section{Introduction}

Starting with the work of Morrey [32], the concept of quasiconvexity has been fundamental in the study of the relaxation of vectorial problems in the calculus of variations, see for example [18, 34, 39]. In particular, if $W: \mathbb{R}^{n \times m} \rightarrow$ $\mathbb{R}$ is continuous and has $p$-growth then the relaxation of the functional $I$ : $W^{1, p}\left(\Omega ; \mathbb{R}^{m}\right) \rightarrow \mathbb{R}$

$$
I[u]=\int_{\Omega} W(D u) d x
$$

is given by

$$
I^{*}[u]=\int_{\Omega} W^{\mathrm{qc}}(D u) d x,
$$

where $W^{\text {qc }}$ is the quasiconvex envelope of $W$, see [32, 1, 18]. Here $\Omega \subset \mathbb{R}^{n}$ is a bounded Lipschitz set, $W^{\mathrm{qc}}$ is defined by (1.3) below. The computation of $W^{\mathrm{qc}}$ is in general difficult, but it was performed in a number of special cases with high symmetry, see for example [20, 17, 41, 16. The key strategy is to construct specific test functions using lamination and rank-one convexity to prove an upper bound, and then to show that the resulting expression is polyconvex, which delivers the lower bound.

One of the main applications of the vectorial calculus of variations is nonlinear elasticity with $m=n$. The physical constraint of non-interpenetration 
of matter leads naturally to the requirement of injectivity of the deformation $u$, a complex nonlocal condition which is often replaced by the simpler condition that $\operatorname{det} D u>0$ almost everywhere. Correspondingly, one assumes that the energy density $W$ diverges when the determinant of the argument is positive and tends to zero. Such energy densities are not continuous on $\mathbb{R}^{n \times n}$ and do not have $p$-growth from above for any $p$, hence the general relaxation theorem is not applicable. Starting with the work of Ball [2] a large body of work developed with the aim of proving existence of minimizers for variational problems with the constraint $\operatorname{det} D u>0$, mainly building upon the concept of polyconvexity. In contrast, to the best of our knowledge, there is no physically-relevant functional incorporating the nonlinear constraint det $D u>0$ for which a nontrivial relaxation is known.

The significance of the constraint $\operatorname{det} D u>0$ depends dramatically on the growth exponent $p$ of the energy, and two main regimes emerge. If $p \geq n$ the deformation $u$ is necessarily continuous. This follows from the Sobolev embedding theorem for $p>n$ and in the case $p=n$ from the work of Vodop'janov and Gol'dštel̆ [43], see also [42]. Further, if a sequence $u_{j}$ converges weakly to some $u$ in $W^{1, n}$ and $\operatorname{det} D u_{j}>0$ almost everywhere, then necessarily $\operatorname{det} D u \geq 0$ almost everywhere. For $p>n$ this follows directly from the properties of the determinant, which in particular give $\operatorname{det} D u_{j} \rightarrow \operatorname{det} D u$ in $L^{1}$ [2, Cor. 6.2.2], and still holds for $p=n$, see [8, Th. 4.1(ii)]. A related treatment with the distributional determinant instead of the pointwise determinant is still possible if $p>n-1$ and a generalized invertibility condition is used instead of $\operatorname{det} D u>0$ [36, see also [35, 14, 23, 25] for subsequent developments.

The case $p<n$ is substantially different, since the deformations are not continuous and can develop holes, as was first shown by Ball [4]. Correspondingly, the constraint of having positive determinant does not pass to the limit and the relaxed problem has a substantially different structure, see for example [8, 28, 27] for further developments. Relaxation in a related situation in which the constraints are lost after rank-one convexification was discussed in [10]. We shall not discuss these cases further here.

The proof of the classical relaxation theorem for continuous integrands is based on a truncation procedure in which one replaces a sequence $u_{j} \in$ $W^{1, p}$ which converges weakly to an affine function by a sequence with the same weak limit, the same energy, and which is affine on the boundary. It is currently unknown if a similar construction can be done if a constraint on the determinant has to be preserved. In two spatial dimensions and under the assumption that $u$ is bilipschitz a solution was given in [11] building upon an involved construction of bilipschitz extensions by Daneri and Pratelli [19]. The approximation of Hölder-continuous homeomorphisms was obtained in [9]. The situation with Sobolev functions is substantially more complex, and was up to now only solved in the case $p=n=2$, see [26]. A related problematic arises in the relaxation of problems with mixed growth, see for example [29]. 
In this paper we prove that the functional (1.2) gives an upper bound on the relaxation of (1.1) for a class of energy densities which are infinite on matrices $F$ with $\operatorname{det} F \leq 0$ and have $p$-growth for some $p \geq 1$ on the set $\{\operatorname{det} F>0\}$, see Theorem 2.1 below. If $W^{\mathrm{qc}}$ is polyconvex and $p \geq n$ then (1.2) coincides with the relaxation of (1.1), see Theorem 2.2 below. The growth assumption can be somewhat relaxed if a suitable integrability of the cofactor is assumed, see Remark 2.3. Since all known explicit quasiconvex envelopes $W^{\text {qc }}$ are quasiconvex, our result fully characterizes the relaxation in all cases where $W^{\mathrm{qc}}$ has been computed and the pointwise determinant constraint survives the relaxation. We also show that our results can be generalized to problems where $\operatorname{det} D u=1$ almost everywhere, see Section 2.2 below.

Notation: We denote by $\mathbb{R}_{+}^{n \times n}=\left\{F \in \mathbb{R}^{n \times n}: \operatorname{det} F>0\right\}$ the set of orientation-preserving matrices, by $B\left(r, x_{0}\right)$ the open ball in $\mathbb{R}^{n}$ and set $B_{r}=$ $B(r, 0)$. Finally $f_{E} f d x$ denotes the mean value of $f$ on $E$.

We define the quasiconvex envelope $W^{\mathrm{qc}}: \mathbb{R}^{n \times n} \rightarrow[0, \infty]$ of a Borelmeasurable function $W: \mathbb{R}^{n \times n} \rightarrow[0, \infty]$ by

$$
W^{\mathrm{qc}}(F)=\inf \left\{f_{B_{1}} W(D \varphi) d x: \varphi \in W^{1, \infty}\left(B_{1} ; \mathbb{R}^{n}\right), \varphi(x)=F x \text { for } x \in \partial B_{1}\right\}
$$

This is not necessarily the same as the largest finite-valued quasiconvex function below $W$, see Remark 3.5 below.

We say that a function $f: \mathbb{R}^{n \times n} \rightarrow[0, \infty]$ is polyconvex if there is a lower semicontinuous and convex function $g: \mathbb{R}^{\tau(n)} \rightarrow[0, \infty]$ such that $f(F)=$ $g(M(F))$, where $M(F)$ denotes all minors of $F$ [2, 18, 34]. In particular, if $n=2$ then $M(F)=(F, \operatorname{det} F)$, if $n=3$ then $M(F)=(F, \operatorname{cof} F, \operatorname{det} F)$. The requirement of lower semicontinuity of $g$ is often not included in the definition but instead enforced through appropriate growth conditions. For finite-valued functions this makes no difference, but for extended-valued we believe the present one to be the definition more naturally related to lower semicontinuity, as the example $g(\operatorname{det} F)=0$ if $\operatorname{det} F>0, g(\operatorname{det} F)=\infty$ otherwise, with the sequence $u(x)=x / j$ shows, see also the discussion in [31].

\section{Main results}

\subsection{Relaxation of orientation-preserving models}

The main result of the paper is a relaxation theorem for coercive variational problems in nonlinear elasticity incorporating a constraint on the determinant, see Theorem 2.2 below. Our key new contribution is a construction for the upper bound which preserves the positive-determinant constraint and leads to the following statement. 
Theorem 2.1. Let $W \in C^{0}\left(\mathbb{R}_{+}^{n \times n},[0, \infty)\right)$ obey

$$
\frac{1}{c}|F|^{p}+\frac{1}{c} \theta(\operatorname{det} F)-c \leq W(F) \leq c|F|^{p}+c \theta(\operatorname{det} F)+c
$$

for some $p \geq 1$ and $c>0$, where $\theta:(0, \infty) \rightarrow[0, \infty)$ is convex and satisfies

$$
\theta(x y) \leq c(1+\theta(x))(1+\theta(y)) \text { for all } x, y \in(0, \infty),
$$

and extend $W$ to $\mathbb{R}^{n \times n}$ by $W(F)=\infty$ if $\operatorname{det} F \leq 0$. Let $W^{\mathrm{qc}}$ be defined as in (1.3), $\Omega \subset \mathbb{R}^{n}$ open, bounded and Lipschitz. For any $u \in W^{1, p}\left(\Omega ; \mathbb{R}^{n}\right)$ there is a sequence $u_{j} \in W^{1, p}\left(\Omega ; \mathbb{R}^{n}\right)$ which converges weakly to $u$ such that $u_{j}-u \in W_{0}^{1, p}$ for all $j$ and

$$
\limsup _{j \rightarrow \infty} \int_{\Omega} W\left(D u_{j}\right) d x \leq \int_{\Omega} W^{\mathrm{qc}}(D u) d x .
$$

Proof. If det $D u>0$ almost everywhere the statement follows from Lemma 3.2 in Section 3 below. From the definition one immediately obtains $W^{\mathrm{qc}}(F)=\infty$ if $\operatorname{det} F \leq 0$, therefore in the other case a constant sequence will do.

If the coercivity exponent $p$ is at least $n$, then the determinant is an $L^{1}$ function and weakly continuous in compact subsets [33], therefore the constraint on the determinant passes to the limit. Complementing Theorem 2.1 with existing compactness and lower semicontinuity results, based on the concept of polyconvexity [2], leads to a full relaxation and existence statement.

Theorem 2.2. Let $W \in C^{0}\left(\mathbb{R}_{+}^{n \times n},[0, \infty)\right)$ obey (2.1 2.2) with $p \geq n$ and

$$
\lim _{t \rightarrow 0} \theta(t)=\infty,
$$

and extend $W$ by $W(F)=\infty$ to the set $\{\operatorname{det} F \leq 0\}$. Let $\Omega \subset \mathbb{R}^{n}$ be an open, bounded, Lipschitz, connected set,

$$
X=\left\{u \in W^{1, p}\left(\Omega ; \mathbb{R}^{n}\right): \operatorname{det} D u>0 \text { a.e. }\right\},
$$

and $f \in C^{0}\left(\mathbb{R}^{n}\right)$ with $|f(t)| \leq c\left(1+|t|^{q}\right)$ for some $q \in[0, p)$. We define $W^{\text {qc }}$ as in (1.3) and the functionals $E, E^{*}: L^{1}\left(\Omega ; \mathbb{R}^{n}\right) \rightarrow \mathbb{R} \cup\{\infty\}$ by

$$
E[u]=\int_{\Omega}(W(D u)+f(u)) d x \text { and } E^{*}[u]=\int_{\Omega}\left(W^{\mathrm{qc}}(D u)+f(u)\right) d x
$$

for $u \in X$, and $E=E^{*}=\infty$ on $L^{1} \backslash X$. Finally assume that $W^{\mathrm{qc}}=W^{\mathrm{pc}}$. Then the following assertions hold:

(i) $E^{*}$ is the relaxation of $E$ with respect to strong $L^{1}$ convergence, in the sense that

$$
E^{*}[u]=\inf \left\{\liminf _{j \rightarrow \infty} E\left[u_{j}\right]: u_{j} \in L^{1}\left(\Omega ; \mathbb{R}^{n}\right), u_{j} \rightarrow u \text { in } L^{1}\right\} .
$$


(ii) The same holds if, for any given relatively open set $\Gamma_{D} \subset \partial \Omega$ and $u_{0} \in$ $X$, the functionals $E$ and $E^{*}$ are set to be $\infty$ outside

$$
\widetilde{X}=X \cap\left\{u=u_{0} \text { on } \Gamma_{D}\right\} .
$$

(iii) The functional $E^{*}$ has a minimizer in the space $\widetilde{X}$.

Proof. By Theorem 2.1 for any $u \in X$ there is $\left(u_{j}\right)_{j \in \mathbb{N}} \subset X$ with $u_{j}=u$ on $\partial \Omega, u_{j} \rightarrow u$ for $j \rightarrow \infty$ in $L^{p}$, and $\lim _{\sup _{j \rightarrow \infty}} E\left[u_{j}\right] \leq E^{*}[u]$. This proves the upper bound in both cases.

Let now $\left(u_{j}\right)_{j \in \mathbb{N}}$ be a sequence in $X$ with $E^{*}\left[u_{j}\right] \leq C<\infty$ for all $j$. From (2.1) one immediately obtains $W^{\mathrm{qc}}(F) \geq|F|^{p} / c-c$. The growth condition on $f$ ensures, since $q<p$, that $\int_{\Omega} W^{\text {qc }}(D u) d x \leq C^{\prime}<\infty$ for all $j$. Taking a subsequence we can assume $u_{j} \rightarrow u$ in $W^{1, p}$ for some $u \in W^{1, p}\left(\Omega ; \mathbb{R}^{n}\right)$. By the continuity of the trace, if $u_{j}=u_{0}$ on $\Gamma_{D}$ for all $j$ then $u=u_{0}$ on $\Gamma_{D}$.

In order to show that $u \in X$ it only remains to prove the condition on the determinant. By [33] we have $\operatorname{det} D u_{j} \rightarrow \operatorname{det} D u$ in $L^{1}(K)$ for all $K \subset \subset \Omega$. Since $\theta$ diverges at 0 the weak limit is positive almost everywhere and $u \in X$.

To prove lower semicontinuity we let $W^{\mathrm{qc}}(F)=g(M(F))$, with $g$ convex and lower semicontinuous, and fix a compact set $K \subset \Omega$. As discussed above, we have $\operatorname{det} D u^{j} \rightarrow \operatorname{det} D u$ in $L^{1}(K)$ for all $K \subset \subset \Omega$. The other minors converge also weakly in $L^{1}$, since $u_{j} \rightarrow u$ in $W^{1, p}$ with $p>n-1$. Therefore $M\left(D u^{j}\right) \rightarrow M(D u)$ in $L^{1}(K)$ and using Jensen's inequality and the convexity of $g$ we obtain

$$
\begin{aligned}
\int_{K} W^{\mathrm{qc}}(D u) d x & =\int_{K} g(M(D u)) d x \leq \liminf _{j \rightarrow \infty} \int_{K} g\left(M\left(D u_{j}\right)\right) d x \\
& \leq \liminf _{j \rightarrow \infty} \int_{\Omega} W^{\mathrm{qc}}\left(D u_{j}\right) d x .
\end{aligned}
$$

The term $\int_{\Omega} f(u) d x$ is continuous. Taking the supremum over all compact subsets $K \subset \Omega$ gives $E^{*}[u] \leq \liminf E^{*}\left[u_{j}\right] \leq \liminf E\left[u_{j}\right]$ and concludes the proof.

Remark 2.3. (i) The result of Theorem 2.1 can be extended to functions $W$ which obey

$$
W(F G) \leq c(1+W(F))(1+W(G))
$$

and

$$
\frac{1}{c}|F|-c \leq W(F) \leq c W^{\mathrm{qc}}(F)+c
$$

instead of (2.1) and (2.2). We discuss in Appendix B the required modifications to the proof. 
(ii) Using this generalization, one can extend Theorem 2.2 to a situation in which $W$ obeys (2.3), 2.4) and the growth condition

$$
\frac{1}{c}|F|^{p}+\frac{1}{c}|\operatorname{cof} F|^{q}+\frac{1}{c} \theta(\operatorname{det} F)-c \leq W(F),
$$

corresponding to the spaces $\mathcal{A}_{p, q}$ introduced by Ball [2], see also [42].

(iii) A different picture arises if one instead uses a constraint on the pointwise determinant, with the material becoming substantially softer, see [4, 8] and [28, 27, 38].

\subsection{Relaxation of incompressible models}

We deal with integrands which are defined on the set of volume-preserving matrices $\Sigma=\left\{F \in \mathbb{R}^{n \times n}: \operatorname{det} F=1\right\}$ and which have $p$-growth. In this framework, we prove the following result.

Theorem 2.4. Let $W \in C^{0}(\Sigma,[0, \infty))$ obey

$$
\frac{1}{c}|F|^{p}-c \leq W(F) \leq c|F|^{p}+c
$$

for some $p \geq 1$ and $c>0$, and set $W(F)=\infty$ if $\operatorname{det} F \neq 1$. Let $W^{\mathrm{qc}}$ be defined as in (1.3), $\Omega \subset \mathbb{R}^{n}$ open, bounded and Lipschitz. For any $u \in W^{1, p}\left(\Omega ; \mathbb{R}^{n}\right)$ there is a sequence $u_{j} \in W^{1, p}\left(\Omega ; \mathbb{R}^{n}\right)$ which converges weakly to u such that $u_{j}-u \in W_{0}^{1, p}$ and

$$
\limsup _{j \rightarrow \infty} \int_{\Omega} W\left(D u_{j}\right) d x \leq \int_{\Omega} W^{\mathrm{qc}}\left(D u_{j}\right) d x .
$$

Proof. The statement follows from Lemma 4.3 below.

Also in this case, if coercivity is sufficient a full relaxation statement follows.

Theorem 2.5. Let $W \in C^{0}(\Sigma,[0, \infty))$ obey (2.5) for some $p \geq n$, let $\Omega \subset \mathbb{R}^{n}$ be open bounded, Lipschitz, connected,

$$
X=\left\{u \in W^{1, p}\left(\Omega ; \mathbb{R}^{n}\right): \operatorname{det} D u=1 \text { a.e. }\right\} .
$$

We set $W(F)=\infty$ if $\operatorname{det} F \neq 1$, define $W^{\mathrm{qc}}$ as in (1.3) and, for $f \in C^{0}\left(\mathbb{R}^{n}\right)$ with $|f(t)| \leq c\left(1+|t|^{q}\right)$ for some $q<p$,

$$
E[u]=\int_{\Omega}(W(D u)+f(u)) d x \text { and } E^{*}[u]=\int_{\Omega}\left(W^{\mathrm{qc}}(D u)+f(u)\right) d x
$$

for $u \in X$, and $E=E^{*}=\infty$ on $L^{1} \backslash X$. Finally assume that $W^{\mathrm{qc}}=W^{\mathrm{pc}}$. Then the following assertions hold. 
(i) $E^{*}$ is the relaxation of $E$ with respect to strong $L^{1}$ convergence, in the sense that

$$
E^{*}[u]=\inf \left\{\liminf _{j \rightarrow \infty} E\left[u_{j}\right]: u_{j} \in L^{1}\left(\Omega ; \mathbb{R}^{n}\right), u_{j} \rightarrow u \text { in } L^{1}\right\} .
$$

(ii) The same holds if, for any given relatively open set $\Gamma_{D} \subset \partial \Omega$ and $u_{0} \in$ $X$, the functionals $E$ and $E^{*}$ are set to be $\infty$ outside

$$
\widetilde{X}=X \cap\left\{u=u_{0} \text { on } \Gamma_{D}\right\} .
$$

(iii) The functional $E^{*}$ has a minimizer in the space $\widetilde{X}$.

Proof. The proof is analogous to the proof of Theorem 2.2.

\subsection{Examples}

We first consider the two-well problem in two dimensions, a classical model for microstructure in martensite [6, 7, 12, 5]. Precisely, we define

$$
W_{2 W}(F)=\operatorname{dist}^{2}\left(F, S O(2) U_{1} \cup S O(2) U_{2}\right)+\theta(\operatorname{det} F),
$$

where

$$
U_{1}=\left(\begin{array}{cc}
\lambda & 0 \\
0 & 1 / \lambda
\end{array}\right), \quad U_{2}=\left(\begin{array}{cc}
1 / \lambda & 0 \\
0 & \lambda
\end{array}\right)
$$

for some fixed $\lambda>1$ are the eigenstrains of the two martensitic phases and $\theta \in C^{0}((0, \infty),[0, \infty))$ is a convex function which obeys (2.2) and with $\lim _{t \rightarrow 0} \theta(t)=\infty$, for example $f(t)=(t-1 / t)^{2}$, extended with $\theta=\infty$ on $(-\infty, 0]$. One is then interested in the functional $I_{2 W}: W^{1,2}\left(\Omega ; \mathbb{R}^{2}\right) \rightarrow[0, \infty]$,

$$
I_{2 W}[u]=\int_{\Omega} W_{2 W}(D u) d x .
$$

In [16] it was shown that the quasiconvex envelope of $W_{2 W}$ is given by

$$
W_{2 W}^{\mathrm{qc}}(F)=h(|F v|,|F w|, \operatorname{det}(F))+\theta(\operatorname{det} F),
$$

where $v=\left(e_{1}+e_{1}\right) / \sqrt{2}, w=\left(e_{1}-e_{1}\right) / \sqrt{2}$ and $h$ is defined by

$$
h(x, y, d)=\min _{\xi \in[x, \infty), \eta \in[y, \infty)}\left(\xi^{2}+\eta^{2}+\left|U_{1}\right|^{2}-2 \sqrt{A(\xi, \eta, d)}\right),
$$

with

$$
A(x, y, d)=\left(x^{2}+y^{2}\right) \frac{\left|U_{1}\right|^{2}}{2}+\left(\lambda^{2}-\frac{1}{\lambda^{2}}\right) \sqrt{x^{2} y^{2}-d^{2}}+2 d,
$$


and that $W_{2 W}^{\mathrm{qc}}$ is polyconvex. Theorem 2.2 then shows that the relaxation of $I_{2 W}$ is given by

$$
I_{2 W}^{*}[u]=\int_{\Omega} W_{2 W}^{\mathrm{qc}}(D u) d x .
$$

A corresponding result holds for models with one potential well only, which can be recovered setting $\lambda=1$ in the previous expressions, the quasiconvex envelope is given for example in 40].

A related situation with the incompressibility constraint can be obtained from the study of nematic elastomers [21, 40, 20, 44, 41]. They are composite materials in which a rubber (polymer) matrix is coupled to a nematic liquid crystal. The rubber has entropic elasticity and is usually modeled as incompressible; the ordering of the nematic liquid crystal leads to elongation in the direction of the nematic order parameter. After minimizing out the nematic director, the standard model 44 can be cast in the form

$$
W_{\text {ne }}(F)= \begin{cases}\sum_{i=1}^{n}\left(\frac{\lambda_{i}(F)}{\gamma_{i}}\right)^{p} & \text { if } \operatorname{det} F=1 \\ \infty & \text { if } \operatorname{det} F \neq 1\end{cases}
$$

where $\lambda_{i}(F)$ are the singular values of $F$, i.e., the eigenvalues of $\left(F^{T} F\right)^{1 / 2}$, and $\gamma_{i} \in(0, \infty)$ are material parameters with $\prod_{i=1}^{n} \gamma_{i}=1$.

In two dimensions one can assume $\gamma_{2}=1 / \gamma_{1}>1$ and, taking the natural exponent $p=2$, one can show that 21 ]

$$
W_{\mathrm{ne}}^{\mathrm{qc}}(F)= \begin{cases}\infty & \text { if } \operatorname{det} F \neq 1 \\ 2 & \text { if } \lambda_{2}(F) \leq \gamma_{2} \text { and } \operatorname{det} F=1 \\ W_{\mathrm{ne}}(F) & \text { otherwise. }\end{cases}
$$

Further, the function $W_{\mathrm{ne}}^{\mathrm{qc}}$ is polyconvex 21]. Therefore Theorem 2.4 shows that the relaxation of $I_{\text {ne }}$ is given by $I_{\text {ne }}^{*}$.

In the physically relevant situation $n=3$ a similar expression is analytically known and turns out to be polyconvex for all $p \geq 1$ [20]. Theorem 2.2 then states that $I_{\text {ne }}^{*}$ is the relaxation of $I_{\text {ne }}$ for all $p \geq 3$. This does not include, however, the physically relevant exponent $p=2$. In this case, as noted in the introduction, the variational model with the pointwise constraint on the determinant would predict cavitation under tension. Tensile experiments are however carried out in a regime in which cavitation does not occur, possibly due to metastability or to additional energy contributions not modeled by $W_{\text {ne }}$ [44]. Indeed, numerical simulations based on the quasiconvex envelope $W_{\mathrm{ne}}^{\mathrm{qc}}$ and excluding cavitation via the choice of a the finite-element space of continuous function lead to good agreement with experimental observations [15. 


\subsection{Strategy of the proof}

The "classical" construction of the upper bound for relaxation theorems is based on density and interpolation. Given a function $u \in W^{1, p}$ one first approximates it strongly in $W^{1, p}$ by a piecewise affine function and then uses a "good" test function $\varphi$ in each of the pieces where $u$ is affine. In doing this it is important that the energy is continuous along the approximating sequence. In our case the energy is infinite on all deformations which are not orientation-preserving, therefore one would need to approximate orientationpreserving $W^{1, p}$ maps with piecewise affine orientation-preserving maps, a problem which, as we discussed above, is very difficult and in general unsolved.

Here we show that the passage through piecewise affine maps is not needed. The key idea is to locally use the composition of the limiting map $u$ with the map with oscillating gradient $\varphi$. Taking the composition preserves the conditions on the determinant, and the map $u \circ \varphi$ belongs to $W^{1, p}$ since $u \in W^{1, p}$ and $\varphi$ is Lipschitz. The key point is to prove that the unrelaxed energy of the composition is close to the relaxed energy of $u$.

For simplicity we focus here on a small ball $B$ where $D u$ is close to the identity (after a change of variables this is true around any Lebesgue point of $D u)$. Let $\varphi \in W^{1, \infty}\left(B, \mathbb{R}^{n}\right)$ be a map from (1.3), which in particular is the identity on the boundary of $B$. The key idea is to define $z=u \circ \varphi$, so that $D z=D u \circ \varphi D \varphi$. If $D u$ is close to the identity and $D z$ is close to $D \varphi$ one then (heuristically) obtains

$$
\int_{B} W(D z) d x \sim \int_{B} W(D \varphi) d x \sim|B| W^{\mathrm{qc}}(\mathrm{Id}) d x \sim \int_{B} W^{\mathrm{qc}}(D u) d x .
$$

This estimate can be made precise if $D u$ is uniformly close to the identity and $W$ is uniformly continuous. Both properties are true only in appropriate subsets, and hence the main part of this work is devoted to the treatment of the exceptional sets, under the assumption that $\|D u-\mathrm{Id}\|_{L^{p}(B)}$ is small.

In the incompressible case, since $\varphi$ is a volume-preserving bilipschitz map a change of variables shows that the smallness of $D u$ - Id in $L^{p}$ immediately translates into the smallness of $(D u-\mathrm{Id}) \circ \varphi$ in $L^{p}$ and hence of the contribution of the set where $D z-D \varphi$ is large. This renders the volumepreserving construction in Lemma 4.2 simpler than the corresponding one in the orientation-preserving case.

In the orientation-preserving case the situation is more complex, since a factor det $D \varphi$ arises from the change of variables formula. One of the problematic terms is

$$
\int_{\{|D u-\mathrm{Id}| \circ \varphi>\varepsilon\}}|D u-\mathrm{Id}|^{p}(\varphi(x)) d x=\int_{\{|D u-\mathrm{Id}|>\varepsilon\}} \frac{|D u-\mathrm{Id}|^{p}(y)}{\operatorname{det} D \varphi(y)} d y .
$$

The fact that $\varphi$ is Lipschitz provides a bound on $\operatorname{det} D \varphi$ but not on its inverse. However, by the very same change of variables formula we know that 
$1 / \operatorname{det} D \varphi \in L^{1}$, therefore the above expression has the form of the integral of the product of two $L^{1}$ functions. This is in general not defined, but can be controlled if one of the two functions is first shifted by an "appropriate amount", as done for example when taking the convolution of two $L^{1}$ functions. Precisely, this means that for every choice of $f, g \in L^{1}\left(B_{1}\right)$ there are many $a_{0} \in B_{1 / 2}$ such that

$$
\int_{B_{1 / 2}} f(x) g\left(x-a_{0}\right) d x \leq\|f\|_{L^{1}\left(B_{1}\right)}\|g\|_{L^{1}\left(B_{1}\right)},
$$

see Lemma 3.1 below for details.

In both cases the local construction is then extended to a global one by a covering argument, see Lemma 3.3 below.

\section{Construction of orientation-preserving maps}

\subsection{Local construction}

Before presenting the construction we show how the translation is exploited. We focus here on the derivation of the estimates, and address measurability and weak differentiability issues in Appendix A.

Lemma 3.1. Let $\psi \in W^{1, \infty}\left(B_{r} ; \overline{B_{r}}\right), g \in L^{1}\left(B_{r}\right), f \in L^{1}\left(B\left(x_{0}, 2 r\right)\right)$ for some $x_{0} \in \mathbb{R}^{n}, r>0$. Then there exists a measurable set $E \subset B\left(x_{0}, r\right)$ of positive $\mathcal{L}^{n}$ measure with the following property. For $a_{0} \in E$ the function

$$
\widetilde{f}(x)=f\left(\psi\left(x-a_{0}\right)+a_{0}\right) g\left(x-a_{0}\right)
$$

belongs to $L^{1}\left(B\left(a_{0}, r\right)\right)$ with

$$
\|\widetilde{f}\|_{L^{1}\left(B\left(a_{0}, r\right)\right)} \leq \frac{1}{\left|B_{r}\right|}\|f\|_{L^{1}\left(B\left(x_{0}, 2 r\right)\right)}\|g\|_{L^{1}\left(B_{r}\right)} .
$$

Proof. We can assume without loss of generality that $f, g \geq 0$. The function $\left(x, a_{0}\right) \mapsto \widetilde{f}(x)$ is $\mathcal{L}^{2 n}$-measurable by Lemma A.1. We define $h: B\left(x_{0}, r\right) \rightarrow$ $\mathbb{R} \cup\{\infty\}$ by

$$
h\left(a_{0}\right)=\int_{B\left(a_{0}, r\right)} \widetilde{f}(x) d x=\int_{B\left(a_{0}, r\right)} f\left(\psi\left(x-a_{0}\right)+a_{0}\right) g\left(x-a_{0}\right) d x
$$

and change variables to

$$
h\left(a_{0}\right)=\int_{B_{r}} f\left(\psi\left(x^{\prime}\right)+a_{0}\right) g\left(x^{\prime}\right) d x^{\prime} .
$$


We integrate over all $a_{0} \in B\left(x_{0}, r\right)$ and interchange the order of integration to obtain

$$
\begin{aligned}
\int_{B\left(x_{0}, r\right)} h\left(a_{0}\right) d a_{0} & =\int_{B_{r}}\left(\int_{B\left(x_{0}, r\right)} f\left(\psi\left(x^{\prime}\right)+a_{0}\right) g\left(x^{\prime}\right) d a_{0}\right) d x^{\prime} \\
& \leq\|f\|_{L^{1}\left(B\left(x_{0}, 2 r\right)\right)}\|g\|_{L^{1}\left(B_{r}\right)} .
\end{aligned}
$$

To conclude we observe that $h$ cannot be almost everywhere larger than its average.

Lemma 3.2. Assume that the function $W \in C^{0}\left(\mathbb{R}_{+}^{n \times n},[0, \infty)\right)$ satisfies the growth condition (2.1) with $p \geq 1$ and the structure condition (2.2) and fix $F \in \mathbb{R}_{+}^{n \times n}$ and $\eta>0$. Then there is $\delta>0$ such that for any $B=B\left(x_{0}, r\right)$ and $u \in W^{1, p}\left(B, \mathbb{R}^{n}\right)$ with

$$
f_{B}\left(|D u-F|^{p}+|\theta(\operatorname{det} D u)-\theta(\operatorname{det} F)|\right) d x \leq \delta \text { and } \operatorname{det} D u>0 \text { a.e. }
$$

there are $a_{0} \in B\left(x_{0}, r / 2\right)$ and $z \in W^{1, p}\left(B, \mathbb{R}^{n}\right)$ with $\operatorname{det} D z>0$ a.e., $z=u$ on $B\left(x_{0}, r\right) \backslash B\left(a_{0}, r / 2\right)$ and

$$
\int_{B\left(a_{0}, r / 2\right)} W(D z) d x \leq \int_{B\left(a_{0}, r / 2\right)}\left(W^{\mathrm{qc}}(D u)+\eta\right) d x .
$$

Additionally,

$$
\int_{B}|u-z|^{p} d x \leq c r^{p} \int_{B}\left(W^{\mathrm{qc}}(D u)+1\right) d x .
$$

If $u$ is Lipschitz, then the same is true for $z$.

Proof. The $L^{p}$ bound follows from the bound on $W(D z)$ using Poincaré and the growth condition, hence we only need to prove (3.2).

By the definition of $W^{\mathrm{qc}}(F)$ there is $\varphi_{\eta} \in W^{1, \infty}\left(B_{r / 2}, \mathbb{R}^{n}\right)$ such that $\varphi_{\eta}(x)=F x$ on $\partial B_{r / 2}$ and

$$
f_{B_{r / 2}} W\left(D \varphi_{\eta}\right) d x \leq W^{\mathrm{qc}}(F)+\eta .
$$

By the growth condition (2.1) we have $\theta\left(\operatorname{det} D \varphi_{\eta}\right) \in L^{1}\left(B_{r / 2}\right)$, and with (2.2) also $\theta\left(\operatorname{det}\left(F^{-1} D \varphi_{\eta}\right)\right) \in L^{1}\left(B_{r / 2}\right)$. Since $\operatorname{det} D \varphi_{\eta}>0$ almost everywhere there is $\gamma>0$ (depending on $F$ and $\eta$ ) such that

$$
\begin{aligned}
\int_{B_{r / 2}} \cap\left\{\operatorname{det} D \varphi_{\eta}<\gamma\right\} & \left(1+\theta\left(\operatorname{det}\left(F^{-1} D \varphi_{\eta}\right)\right)\right) d x \\
\leq & \frac{\left|B_{r / 2}\right| \eta}{\left(1+\left\|F^{-1} D \varphi_{\eta}\right\|_{L^{\infty}}^{p}\right)\left(1+|F|^{p}+\theta(\operatorname{det} F)\right)} .
\end{aligned}
$$


The choice of the constant on the right-hand side will become clear after (3.11).

The function $F^{-1} \varphi_{\eta}$ is Lipschitz continuous and therefore, by [3, Theorem 1], $F^{-1} \varphi_{\eta}\left(B_{r / 2}\right) \subset \overline{B_{r / 2}}$. For some $a_{0} \in B\left(x_{0}, r / 2\right)$ chosen below, we construct the function $z: B=B\left(x_{0}, r\right) \rightarrow \mathbb{R}^{n}$ by

$$
z(x)= \begin{cases}u\left(F^{-1} \varphi_{\eta}\left(x-a_{0}\right)+a_{0}\right) & \text { if } x \in B^{\prime}=B\left(a_{0}, r / 2\right), \\ u(x) & \text { otherwise. }\end{cases}
$$

By Lemma A.2 (with $\psi=F^{-1} \varphi_{\eta}$ ), there exists a null set $N$ such for all choices of $a_{0} \notin N$ the first expression belongs to $W^{1,1}$. Further we can compute its weak derivative by the usual chain rule, and the traces on $\partial B^{\prime}$ of the two expressions coincide. In particular, $z \in W^{1,1}\left(B^{\prime} ; \mathbb{R}^{n}\right)$. In order to obtain an estimate on the derivative we choose $a_{0} \in E \backslash N$ via Lemma 3.1. applied to the ball $B$ with $f=|D u-F|^{p}+|\theta(\operatorname{det} D u)-\theta(\operatorname{det} F)|$ and $g=1+\theta\left(\operatorname{det}\left(F^{-1} D \varphi_{\eta}\right)\right)$. Then

$$
f_{B^{\prime}}(1+\theta(\operatorname{det} D v))\left(|D u-F|^{p}+|\theta(\operatorname{det} D u)-\theta(\operatorname{det} F)|\right) \circ v d x \leq c_{\eta} \delta,
$$

where $v(x)=F^{-1} \varphi_{\eta}\left(x-a_{0}\right)+a_{0}$ and

$$
c_{\eta}=2^{n} f_{B_{r / 2}}\left(1+\theta\left(\operatorname{det} F^{-1} D \varphi_{\eta}\right)\right) d x
$$

(since $W$ and $F$ are fixed for the entire proof, we emphasize the dependence of the constants on $\eta$ ). For the rest of the proof we only need to deal with the fixed inner ball $B^{\prime}$.

Let $R_{\eta}=\|D v\|_{L^{\infty}}, M_{\eta}=\left\|D \varphi_{\eta}\right\|_{L^{\infty}}$. Since $W$ is continuous in $\mathbb{R}_{+}^{n \times n}$ there is $\varepsilon \in(0,1)$ such that

$$
\begin{array}{r}
|W(\xi)-W(\zeta)| \leq \eta \text { for all } \xi, \zeta \in \mathbb{R}_{+}^{n \times n} \text { with }|\zeta| \leq M_{\eta}, \\
\operatorname{det} \zeta \geq \gamma \text { and }|\xi-\zeta| \leq \varepsilon R_{\eta},
\end{array}
$$

where $\gamma$ was defined in (3.4) and depends only on $W, F$ and $\eta$. Moreover, $W^{\text {qc }}$ is continuous in $\mathbb{R}_{+}^{n \times n}$. This is proven for example in [22, Th. 2.4 and Prop. 2.3 by showing that $W^{\mathrm{qc}}$ is rank-one convex, and hence separately convex, in the open set $\mathbb{R}_{+}^{n \times n}$. Hence we may assume additionally that

$$
\left|W^{\mathrm{qc}}(\xi)-W^{\mathrm{qc}}(F)\right|+|\theta(\operatorname{det} \xi)-\theta(\operatorname{det} F)| \leq \eta \text { for all } \xi \text { with }|\xi-F| \leq \varepsilon .
$$

The parameter $\varepsilon$ depends on $\eta$, but not on $u$ and $\delta$. We compute, with $\widehat{\varphi}_{\eta}(x)=\varphi_{\eta}\left(x-a_{0}\right)$,

$$
\begin{aligned}
\int_{B^{\prime}}\left(W(D z)-W^{\mathrm{qc}}(D u)\right) d x= & \int_{B^{\prime}}\left(W(D z)-W\left(D \widehat{\varphi}_{\eta}\right)\right) d x \\
& +\int_{B^{\prime}}\left(W\left(D \widehat{\varphi}_{\eta}\right)-W^{\mathrm{qc}}(F)\right) d x \\
& +\int_{B^{\prime}}\left(W^{\mathrm{qc}}(F)-W^{\mathrm{qc}}(D u)\right) d x
\end{aligned}
$$


and estimate the three terms separately.

The second term in (3.8) is bounded by $\eta\left|B^{\prime}\right|$ by the definition of $\varphi_{\eta}$, see (3.3). To treat the third one we use (3.7) to obtain

$$
W^{\mathrm{qc}}(F) \leq W^{\mathrm{qc}}(D u)+\eta \quad \text { on the set where }|D u-F| \leq \varepsilon .
$$

The complement is small, indeed, from (3.1) we obtain

$$
\begin{aligned}
\int_{B^{\prime}}\left(W^{\mathrm{qc}}(F)-W^{\mathrm{qc}}(D u)\right) d x & \leq \eta\left|B^{\prime}\right|+W^{\mathrm{qc}}(F) \mathcal{L}^{n}(|D u-F|>\varepsilon) \\
& \leq \eta\left|B^{\prime}\right|+W^{\mathrm{qc}}(F) \frac{1}{\varepsilon^{p}}|B| \delta .
\end{aligned}
$$

To estimate the first term in (3.8) we distinguish three subsets: $\omega=B^{\prime} \cap$ $\{|D u-F| \circ v \geq \varepsilon\}, \omega_{d}=B^{\prime} \cap\left\{\operatorname{det} D \widehat{\varphi}_{\eta}<\gamma\right\} \backslash \omega$ and the rest $B^{\prime} \backslash \omega \backslash \omega_{d}$.

In $B^{\prime} \backslash \omega \backslash \omega_{d}$ we have $|D v| \leq R_{\eta}$, $\operatorname{det} D \widehat{\varphi}_{\eta} \geq \gamma,|D u-F| \circ v \leq \varepsilon$ and therefore, since

$$
D z=D u \circ v D v=(D u-F) \circ v D v+D \widehat{\varphi}_{\eta}
$$

we obtain

$$
\left|D z-D \widehat{\varphi}_{\eta}\right| \leq|D u-F| \circ v|D v| \leq \varepsilon R_{\eta} .
$$

By the continuity estimate (3.6) we obtain

$$
\left|W(D z)-W\left(D \widehat{\varphi}_{\eta}\right)\right| \leq \eta
$$

and therefore

$$
\int_{B^{\prime} \backslash \omega_{d} \backslash \omega}\left(W(D z)-W\left(D \widehat{\varphi}_{\eta}\right)\right) d x \leq \eta\left|B^{\prime}\right| .
$$

In the two error sets we use the growth estimate (2.1), which gives

$$
W(D z) \leq c\left(1+|D u|^{p} \circ v|D v|^{p}+\theta(\operatorname{det} D u \circ v \operatorname{det} D v)\right) .
$$

With $|D v| \leq R_{\eta}$ and (2.2) we obtain

$$
W(D z) \leq c\left(1+R_{\eta}^{p}|D u|^{p} \circ v+(1+\theta(\operatorname{det} D u) \circ v)(1+\theta(\operatorname{det} D v)),\right.
$$

where $c$ only depends on $W$. At this point we treat the two error sets separately. For the estimate on $\omega$ we observe that $|D u-F| \geq \varepsilon$ implies

$$
|D u|+1 \leq|D u-F|+|F|+1 \leq\left(\frac{|F|+1}{\varepsilon}+1\right)|D u-F|
$$

and

$$
\theta(\operatorname{det} D u) \leq|\theta(\operatorname{det} D u)-\theta(\operatorname{det} F)|+\frac{\theta(\operatorname{det} F)}{\varepsilon^{p}}|D u-F|^{p}
$$


Therefore (3.10) gives

$$
\begin{aligned}
& \int_{\omega} W(D z) \leq c \int_{\omega}(1+\theta(\operatorname{det} D v))\left(1+R_{\eta}^{p}|D u|^{p}+\theta(\operatorname{det} D u)\right) \circ v d x \\
& \quad \leq c_{\eta} \int_{\omega}(1+\theta(\operatorname{det} D v))\left(|D u-F|^{p}+|\theta(\operatorname{det} D u)-\theta(\operatorname{det} F)|\right) \circ v d x \\
& \quad \leq c_{\eta}\left|B^{\prime}\right| \delta .
\end{aligned}
$$

where in the last step we used (3.5). The constant depends on $W, F$ and $\eta$ (via $\varepsilon$ ), but not on $\delta$ and $u$.

In $\omega_{d}$ instead we have $|D u-F| \circ v \leq \varepsilon$. Then, recalling the continuity estimate (3.7), we have $|D u| \circ v \leq|F|+1$ and $\theta(\operatorname{det} D u \circ v) \leq \theta(\operatorname{det} F)+1$ and therefore (3.10) reduces to

$$
\begin{aligned}
W(D z) & \leq c\left(1+R_{\eta}^{p}\left(1+|F|^{p}\right)+(1+\theta(\operatorname{det} F))(1+\theta(\operatorname{det} D v))\right. \\
& \leq c_{*}\left(1+\left\|F^{-1} D \varphi_{\eta}\right\|_{\infty}^{p}\right)\left(1+|F|^{p}+\theta(\operatorname{det} F)\right)(1+\theta(\operatorname{det} D v)),
\end{aligned}
$$

with a constant $c_{*}>0$ which depends only on $W$. With (3.4) we conclude

$$
\int_{\omega_{d}} W(D z) d x \leq c_{*}\left|B^{\prime}\right| \eta
$$

Adding all terms we obtain

$$
\int_{B^{\prime}}\left(W(D z)-W^{\mathrm{qc}}(D u)\right) d x \leq\left(3 \eta+2^{n} \frac{W^{\mathrm{qc}}(F)}{\varepsilon^{p}} \delta+c_{\eta} \delta+c_{*} \eta\right)\left|B^{\prime}\right| .
$$

Since $c_{*}$ depends only on $W$, choosing $\delta$ sufficiently small the proof is concluded.

\subsection{Upper bound}

Lemma 3.3 (Recovery sequence). Let $\Omega \subset \mathbb{R}^{n}$ open, Lipschitz, bounded, let $W \in C^{0}\left(\mathbb{R}_{+}^{n \times n},[0, \infty)\right)$ obey (2.1 2.2). Then for any $u \in W^{1, p}\left(\Omega ; \mathbb{R}^{n}\right)$ with $\operatorname{det} D u>0$ almost everywhere there is a sequence $u_{j} \rightarrow u$ in $W^{1, p}$ such that $\operatorname{det} D u_{j}>0$ almost everywhere, $u_{j}=u$ on $\partial \Omega$ and

$$
\limsup _{j \rightarrow \infty} \int_{\Omega} W\left(D u_{j}\right) d x \leq \int_{\Omega} W^{\mathrm{qc}}(D u) d x .
$$

If additionally $u \in W^{1, \infty}$ then also $u_{j} \in W^{1, \infty}$.

Proof. Fix $\eta>0$. It suffices to construct $w$ with $\|u-w\|_{p} \leq \eta, w=u$ on $\partial \Omega$, and $\int_{\Omega} W(D w) d x \leq \int_{\Omega} W^{\mathrm{qc}}(D u) d x+\eta$.

If $\int_{\Omega} W^{\mathrm{qc}}(D u) d x=\infty$ the constant sequence will do, hence we can assume that $W^{\mathrm{qc}} \circ D u \in L^{1}$. By convexity of $\theta$ and of the $p$-norm the definition of $W^{\mathrm{qc}}(F)$ gives

$$
\frac{1}{c}|F|^{p}+\frac{1}{c} \theta(\operatorname{det} F)-c \leq W^{\mathrm{qc}}(F) \text { for all } F,
$$


therefore $|D u|^{p}$ and $\theta(\operatorname{det} D u)$ are also integrable. We denote by $E$ the set of Lebesgue points of $D u$ and $\theta(\operatorname{det} D u)$. For every $x \in E$ we set $F(x)=D u(x)$ and choose $\delta(x)$ as in Lemma 3.2 for this $F$ and $\eta$ as above.

The construction is done by successive application of Lemma 3.2. We set $w_{0}=u, \Omega_{0}=\Omega$ and describe how to pass from $\left(w_{j}, \Omega_{j}\right)$ to $\left(w_{j+1}, \Omega_{j+1}\right)$. For all $x \in E \cap \Omega_{j}$ we choose $r_{j}(x) \in(0, \eta)$ such that $B\left(x, r_{j}(x)\right) \subset \Omega_{j}$ and

$$
f_{B(x, r)}\left(\left|D w_{j}-F(x)\right|^{p}+\left|\theta\left(\operatorname{det} D w_{j}\right)-\theta(\operatorname{det} F(x))\right|\right) d x^{\prime} \leq \delta(x)
$$

for all $r<r_{j}(x)$. This gives a fine cover of $E \cap \Omega_{j}$. We extract a disjoint subcover $B\left(x_{k}, r_{k}\right)_{k \in \mathbb{N}}$ and from this subcover finitely many balls $B\left(x_{k}, r_{k}\right)_{k=0, \ldots, M}$ which cover at least half the volume of $\Omega_{j}$.

We set $w_{j+1}=w_{j}$ on $\Omega \backslash \cup_{k=0}^{M} B\left(x_{k}, r_{k}\right)$ and define $w_{j+1}$ as the result of Lemma 3.2 in each of the balls. Then $w_{j+1} \in W^{1, p}\left(\Omega ; \mathbb{R}^{n}\right)$ and $w_{j+1}=w_{j}=u$ on $\partial \Omega$. Further, the smaller balls $B\left(x_{k}^{\prime}, r_{k} / 2\right) \subset B\left(x_{k}, r_{k}\right)$ obey

$$
\int_{B\left(x_{k}^{\prime}, r_{k} / 2\right)} W\left(D w_{j+1}\right) d x \leq \int_{B\left(x_{k}^{\prime}, r_{k} / 2\right)}\left(W^{\mathrm{qc}}(D u)+\eta\right) d x
$$

and

$$
\int_{B\left(x_{k}^{\prime}, r_{k} / 2\right)}\left|w_{j+1}-u\right|^{p} d x \leq c \eta^{p} \int_{B\left(x_{k}^{\prime}, r_{k} / 2\right)}\left(1+W^{\mathrm{qc}}(D u)\right) d x
$$

with $w_{j+1}=w_{j}$ outside these balls. Finally we set $\Omega_{j+1}=\Omega_{j} \backslash \cup_{k=0}^{M} \bar{B}\left(x_{k}^{\prime}, r_{k} / 2\right)$, so that $\left|\Omega_{j+1}\right| \leq\left(1-2^{-n-1}\right)\left|\Omega_{j}\right|$, and iterate. We remark that $w_{j+1}=u$ on the open set $\Omega_{j+1}$, hence there is no need to redefine $E, F$ and $\delta$ at each step. This concludes the construction of the sequence $w_{j}$.

It remains to show that $w_{j}$, for $j$ sufficiently large, has the desired properties. Each of these functions coincides with $u$ outside a finite number of disjoint balls, and has been modified exactly once in each of those balls. By (3.13) we have

$$
\int_{\Omega}\left|w_{j}-u\right|^{p} d x \leq c \eta^{p} \int_{\Omega}\left(1+W^{\mathrm{qc}}(D u)\right) d x
$$

hence $w_{j}$ is close to $u$ in $L^{p}$, independently of $j$.

Analogously from (3.12) we deduce, for the union of the balls $\Omega \backslash \Omega_{j}$,

$$
\int_{\Omega \backslash \Omega_{j}} W\left(D w_{j}\right) d x \leq \int_{\Omega \backslash \Omega_{j}}\left(W^{\mathrm{qc}}(D u)+\eta\right) d x
$$

which implies

$$
\int_{\Omega} W\left(D w_{j}\right) d x \leq \int_{\Omega \backslash \Omega_{j}}\left(W^{\mathrm{qc}}(D u)+\eta\right) d x+\int_{\Omega_{j}} W(D u) d x .
$$


Since $\left|\Omega_{j}\right| \leq\left(1-2^{-n-1}\right)^{j}|\Omega| \rightarrow 0$ and by the growth condition $W(D u) \in$ $L^{1}(\Omega)$, for sufficiently large $j$ we have

$$
\int_{\Omega} W\left(D w_{j}\right) d x \leq \int_{\Omega}\left(W^{\mathrm{qc}}(D u)+2 \eta\right) d x,
$$

as required.

Lemma 3.4 (Quasiconvexity). The function $W^{\mathrm{qc}}$ is quasiconvex.

Remark 3.5. $W^{\mathrm{qc}}$ is the largest (extended-valued) quasiconvex function below $W$, hence in this sense its quasiconvex envelope. This function does not necessarily coincide with the supremum of all finite-valued quasiconvex functions below $W$; in particular, this is not true for the function discussed in [8, Example 3.5].

Proof. Fix $F$ with $\operatorname{det} F>0, \Omega=B_{1}, \psi \in W^{1, \infty}\left(B_{1}, \mathbb{R}^{n}\right)$ with $\psi(x)=F x$ on $\partial B_{1}$. We need to show that

$$
W^{\mathrm{qc}}(F) \leq f_{B_{1}} W^{\mathrm{qc}}(D \psi) d x .
$$

By Lemma 3.3 there is a sequence $\varphi_{j} \in W^{1, \infty}$ with $\varphi_{j}(x)=\psi(x)=F x$ on $\partial B_{1}$ and such that

$$
\limsup _{j \rightarrow \infty} \int_{B_{1}} W\left(D \varphi_{j}\right) d x \leq \int_{B_{1}} W^{\mathrm{qc}}(D \psi) d x .
$$

Since every $\varphi_{j}$ is admissible in the definition of $W^{\mathrm{qc}}(F)$ we obtain

$$
W^{\mathrm{qc}}(F) \leq f_{B_{1}} W\left(D \varphi_{j}\right) d x
$$

for all $j$, and in particular

$$
W^{\mathrm{qc}}(F) \leq f_{B_{1}} W^{\mathrm{qc}}(D \psi) d x
$$

as desired.

\section{Construction of volume-preserving maps}

In this case the translation is not needed, and correspondingly the proof of Lemma 4.2 is simpler than the one of Lemma 3.2, we give it in detail since it illustates in a compact way the key ideas of our constrruction. At the same time the continuity of $W^{\mathrm{qc}}$ is less clear than for orientation-preserving maps. It essentially follows from the results of [37, 13]. Since it was not stated there we briefly show how it can be derived from the construction in [13]. 
Lemma 4.1. Given $W: \Sigma \rightarrow[0, \infty)$, extended by $\infty$ elsewhere, we define $W^{\mathrm{qc}}$ by (1.3). The function $W^{\mathrm{qc}}$ is rank-one convex and hence continuous on $\Sigma$.

Proof. We first observe that, by general scaling and covering arguments, the definition of $W^{\text {qc }}$ does not depend on the domain, and in particular

$$
W^{\mathrm{qc}}(F)=\inf \left\{f_{\omega} W(D \varphi) d x: \varphi \in W^{1, \infty}\left(\omega ; \mathbb{R}^{n}\right), \varphi(x)=F x \text { for } x \in \partial \omega\right\}
$$

for any bounded open nonempty polyhedron $\omega \subset \mathbb{R}^{n}$.

To prove rank-one convexity we fix $A, B \in \Sigma$ with $\operatorname{rank}(A-B)=1$ and $\lambda \in(0,1)$. We define $F=\lambda A+(1-\lambda) B$. By the construction in [13, Th. 2.1] (with $n=m=r, P=Q=\mathrm{Id}, t=1, \varepsilon=1$ ) there is a finite set $K \subset \Sigma$ such that for any $\delta>0$ one can find a polyhedron $\Omega$ and a piecewise affine function $u \in W^{1, \infty}\left(\Omega ; \mathbb{R}^{n}\right)$ such that $u(x)=F x$ on $\partial \Omega, D u \in K \subset \Sigma$ almost everywhere, $|\{D u \notin\{A, B\}\}| \leq \delta|\Omega|$. The latter, together with the boundary data, implies

$$
|\{D u=A\}| \leq(\lambda+c \delta)|\Omega| \text { and }|\{D u=B\}| \leq(1-\lambda+c \delta)|\Omega|,
$$

with $c$ depending on $A$ and $B$. Further, the set $\{D u=A\}$ is a finite union of simplexes $\omega_{j}^{A}$. For each of them there is, by (4.1) with $F=A$, a Lipschitz function $v_{j}^{A}$ with $v_{j}^{A}=u$ on $\partial \omega_{j}^{A}$ and

$$
\int_{\omega_{j}^{A}} W\left(D v_{j}^{A}\right) d x \leq\left|\omega_{j}^{A}\right|\left(W^{\mathrm{qc}}(A)+\delta\right) .
$$

The same holds for the set $\{D u=B\}$. We set $w=v_{j}^{A}$ on each $\omega_{j}^{A}, w=v_{j}^{B}$ on each $\omega_{j}^{B}, w=u$ on the rest. Since $w(x)=F x$ on $\partial \Omega$ we have

$$
\begin{aligned}
W^{\mathrm{qc}}(F) \leq & f_{\Omega} W(D w) d x \leq \frac{|\{D u=A\}|}{|\Omega|}\left(W^{\mathrm{qc}}(A)+\delta\right) \\
& +\frac{|\{D u=B\}|}{|\Omega|}\left(W^{\mathrm{qc}}(B)+\delta\right)+\frac{|\{D u \notin\{A, B\}\}|}{|\Omega|} \max W(K) \\
& \leq \lambda W^{\mathrm{qc}}(A)+(1-\lambda) W^{\mathrm{qc}}(B)+c \delta \max W(K),
\end{aligned}
$$

with $c$ depending on $A$ and $B$. Taking $\delta \rightarrow 0$ (with fixed $K$ ) this implies the desired inequality $W^{\mathrm{qc}}(F) \leq \lambda W^{\mathrm{qc}}(A)+(1-\lambda) W^{\mathrm{qc}}(B)$. Since $W^{\mathrm{qc}}$ is rankone convex, it is separately convex in suitable variables and hence continuous (for details see, e.g., [13, Step 2 in the proof of Th. 3.1]).

Lemma 4.2. Let $W \in C^{0}(\Sigma ;[0, \infty))$ obey (2.5) for some $p \geq 1$. Then for any $F \in \mathbb{R}^{n \times n}$ and $\eta>0$ there is $\delta>0$ such that the following holds: For any ball $B=B\left(x_{0}, r\right)$ and any function $u \in W^{1, p}\left(B, \mathbb{R}^{n}\right)$ with

$$
f_{B}|D u-F|^{p} d x \leq \delta \text { and } D u \in \Sigma \text { a.e. }
$$


one can find $z \in W^{1, p}\left(B, \mathbb{R}^{n}\right)$ with $u=z$ on $\partial B$,

$$
f_{B} W(D z) d x \leq f_{B}\left(W^{\mathrm{qc}}(D u)+\eta\right) d x \text { and } D z \in \Sigma \text { a.e. }
$$

Additionally,

$$
f_{B}|u-z|^{p} d x \leq c r^{p} f_{B}\left(1+W^{\mathrm{qc}}(D u)\right) d x .
$$

Proof. Let $\varphi_{\eta} \in W^{1, \infty}\left(B, \mathbb{R}^{n}\right)$ be such that $\varphi_{\eta}(x)=F x$ on $\partial B$ and

$$
f_{B} W\left(D \varphi_{\eta}\right) d x \leq W^{\mathrm{qc}}(F)+\eta
$$

We define

$$
v=F^{-1} \varphi_{\eta}
$$

and observe that, by [3, Theorem 2], $v$ is a bilipschitz map from $B$ onto itself. Therefore we can define

$$
z=u \circ v \in W^{1, p}\left(B, \mathbb{R}^{n}\right)
$$

and compute its gradient

$$
D z=D u \circ v D v=(D u-F) \circ v D v+D \varphi_{\eta} .
$$

We set $R_{\eta}=\|D v\|_{\infty}, M_{\eta}=\left\|D \varphi_{\eta}\right\|_{\infty}$ and choose $\varepsilon \in(0,1)$ such that

$$
|W(\xi)-W(\zeta)| \leq \eta \text { whenever }|\zeta| \leq M_{\eta} \text { and }|\xi-\zeta| \leq \varepsilon R_{\eta}
$$

and

$$
\left|W^{\mathrm{qc}}(\xi)-W^{\mathrm{qc}}(F)\right| \leq \eta \text { whenever }|\xi-F| \leq \varepsilon .
$$

In order to estimate the integral

$$
\begin{aligned}
\int_{B}\left(W(D z)-W^{\mathrm{qc}}(D u)\right) d x= & \int_{B}\left(W(D z)-W\left(D \varphi_{\eta}\right)\right) d x \\
& +\int_{B}\left(W\left(D \varphi_{\eta}\right)-W^{\mathrm{qc}}(F)\right) d x \\
& +\int_{B}\left(W^{\mathrm{qc}}(F)-W^{\mathrm{qc}}(D u)\right) d x
\end{aligned}
$$

we consider the three terms separately. The second integral in (4.4) is bounded by $\eta|B|$ by the definition of $\varphi_{\eta}$. In order to estimate the last integral in (4.4) we use (4.3) to obtain

$$
W^{\mathrm{qc}}(F) \leq W^{\mathrm{qc}}(D u)+\eta \quad \text { on the set where }|D u-F| \leq \varepsilon .
$$


The complement is small and gives a small contribution. Precisely,

$$
\begin{aligned}
\int_{B}\left(W^{\mathrm{qc}}(F)-W^{\mathrm{qc}}(D u)\right) d x & \leq \eta|B|+W^{\mathrm{qc}}(F) \mathcal{L}^{n}(|D u-F|>\varepsilon) \\
& \leq \eta|B|+W^{\mathrm{qc}}(F) \frac{1}{\varepsilon^{p}}|B| \delta .
\end{aligned}
$$

In order to estimate the first integral in (4.4) we distinguish the set $\omega=$ $\{|D u-F| \circ v>\varepsilon\}$ and the rest. On $B \backslash \omega$, from the explicit expression for $D z$ we obtain

$$
\left|D z-D \varphi_{\eta}\right| \leq\|D v\|_{\infty}|D u-F| \circ v \leq \varepsilon R_{\eta}
$$

and recalling (4.2) we can estimate

$$
\left|W(D z)-W\left(D \varphi_{\eta}\right)\right| \leq \eta \text { on } B \backslash \omega .
$$

Since $|D u-F| \geq \varepsilon$ implies

$$
|D u|+1 \leq|D u-F|+|F|+1 \leq\left(\frac{|F|+1}{\varepsilon}+1\right)|D u-F|,
$$

the contribution of $\omega$ can be estimated by

$$
\int_{\omega} W(D z) d x \leq c \int_{\omega}\left(R_{\eta}^{p}|D u|^{p} \circ v+1\right) d x \leq c_{F, \varepsilon} \int_{\omega}|D u-F|^{p} \circ v d x
$$

where the constant depends on $F, \eta$ and $\varepsilon$. Finally, $v$ is a bilipschitz map from $B$ onto itself with $\operatorname{det} D v=1$ almost everywhere (see [3, Theorem 2]) and therefore

$$
\int_{\omega} W(D z) d x \leq c_{F, \varepsilon} \int_{B}|D u-F|^{p} \circ v d x=c_{F, \varepsilon} \int_{B}|D u-F|^{p} d x \leq c_{F, \varepsilon} \delta|B| .
$$

Collecting terms we conclude

$$
f_{B}\left(W(D z)-W^{\mathrm{qc}}(D u)\right) d x \leq 3 \eta+\frac{W^{\mathrm{qc}}(F)}{\varepsilon^{p}} \delta+c_{F, \varepsilon} \delta .
$$

Since $\varepsilon$ depends on $\eta$ and $F$ but not on $\delta$ and $u$, choosing $\delta$ sufficiently small the proof is concluded. The $L^{p}$ estimate follows from the growth estimate and Poincaré's inequality.

Lemma 4.3 (Recovery sequence). Let $\Omega \subset \mathbb{R}^{n}$ open, Lipschitz, bounded, $u \in W^{1, p}\left(\Omega ; \mathbb{R}^{n}\right)$ with $D u \in \Sigma$ almost everywhere. Then there is a sequence $u_{j} \rightarrow u$ in $W^{1, p}$ such that $\operatorname{det} D u_{j} \in \Sigma$ almost everywhere and

$$
\limsup _{j \rightarrow \infty} \int W\left(D u_{j}\right) d x \leq \int W^{\mathrm{qc}}(D u) d x .
$$

If additionally $u \in W^{1, \infty}$ then also $u_{j} \in W^{1, \infty}$. 
Proof. The proof is just like the one in Lemma 3.3, for brevity we do not repeat it.

Lemma 4.4 (Quasiconvexity). The function $W^{\mathrm{qc}}$ is quasiconvex.

Proof. The proof is just like the one of Lemma 3.4, for brevity we do not repeat it.

\section{A Composition of Sobolev functions with Lipschitz functions}

The composition of a Lipschitz with a Sobolev function and the composition of a Sobolev with a bilipschitz function are standard. Although there is a substantial literature on the subject, see for example [24, 30] and references therein, we have been unable to find the statement needed here on the composition of a Sobolev with a Lipschitz function, hence we give a short selfcontained proof. To see the difficulty with measurability one can consider the example $\psi\left(x_{1}, x_{2}\right)=\left(x_{1}, 0\right), f\left(x_{1}, x_{2}\right)=h\left(x_{1}\right) \chi_{\{0\}}\left(x_{2}\right)$, with $h: \mathbb{R} \rightarrow \mathbb{R}$ not measurable. Then $f=0 \mathcal{L}^{2}$-almost everywhere but $f \circ \psi$ is not measurable. To see the difficulty with integrability one can consider $f(x)=|x|^{-1 / 2}$ in the unit ball of $\mathbb{R}^{2}$, with $\psi(x)=|x| x$ around the origin. Then $f$ is in $W^{1,1}$ but $(f \circ \psi)(x)=|x|^{-1}$ is not.

Lemma A.1. Let $\psi \in W^{1, \infty}\left(B_{1} ; \bar{B}_{1}\right), f_{k} \in L^{1}\left(B_{2} ; \mathbb{R}^{m}\right)$ with $\sum_{k}\left\|f_{k}\right\|_{L^{1}\left(B_{2}\right)}<$ $\infty$. Then the maps $\left(x, a_{0}\right) \mapsto f_{k}\left(a_{0}+\psi\left(x-a_{0}\right)\right)$ are $\mathcal{L}^{2 n}$ measurable and for almost all $a_{0} \in B_{1}$ the functions

$$
z_{k}(x)=f_{k}\left(a_{0}+\psi\left(x-a_{0}\right)\right)
$$

are in $L^{1}\left(B\left(a_{0}, 1\right)\right)$ with $\sum_{k}\left\|z_{k}\right\|_{L^{1}\left(B\left(a_{0}, 1\right)\right)}<\infty$.

Proof. We define the continuous function $g: B_{1} \times B_{1} \rightarrow B_{2}$ by $g(x, y)=$ $x+\psi(y)$ and show that for any $k$ the function $f_{k} \circ g$ is $\mathcal{L}^{2 n}$-measurable.

Let $A \subset \mathbb{R}^{m}$ be open. Then $f_{k}^{-1}(A) \subset B_{2}$ is $\mathcal{L}^{n}$-measurable, therefore $f_{k}^{-1}(A)=E \backslash N$, with $E$ Borel and $N$ a null set. Since $g$ is continuous, $g^{-1}(E)$ is Borel. It remains to show that $|N|=0$ implies $g^{-1}(N)=0$. Let $F \subset B_{2}$ be Borel with $N \subset F$ and $|F|=0$. Then $g^{-1}(F)$ is Borel and $\mathcal{L}^{2 n}$ measurable. For any $y \in \mathbb{R}^{n}$, the set $T_{y}=\left\{x \in \mathbb{R}^{n}: g(x, y) \in F\right\}=F-\psi(y)$ is a $\mathcal{L}^{n}$-null set. By Fubini's theorem

$$
\mathcal{L}^{2 n}\left(g^{-1}(F)\right)=\int_{\mathbb{R}^{n}} \mathcal{L}^{n}\left(T_{y}\right) d y=0 .
$$

Therefore $f_{k} \circ g$ is measurable. A second application of Fubini's theorem shows that for almost all $a_{0} \in B_{1}$ each function $x \mapsto f_{k}\left(g\left(a_{0}, x\right)\right)$ is measurable; 
clearly the same holds for the translations $z_{k}(x)=f_{k}\left(g\left(a_{0}, x-a_{0}\right)\right)$. We conclude that for almost all $a_{0} \in B_{1}$ all the functions $z_{k}$ are $\mathcal{L}^{n}$-measurable.

We define $A: B_{1} \rightarrow[0, \infty]$ by

$$
\begin{aligned}
A\left(a_{0}\right) & =\sum_{k \in \mathbb{N}}\left\|z_{k}\right\|_{L^{1}\left(B\left(a_{0}, 1\right)\right)}=\sum_{k \in \mathbb{N}} \int_{B\left(a_{0}, 1\right)}\left|f_{k}\right|\left(a_{0}+\psi\left(x-a_{0}\right)\right) d x \\
& =\sum_{k \in \mathbb{N}} \int_{B_{1}}\left|f_{k}\right|\left(a_{0}+\psi\left(x^{\prime}\right)\right) d x^{\prime} .
\end{aligned}
$$

The integrand is nonnegative and measurable, hence we can interchange the order of summation and integration. Since the integrand is measurable as a function on $\mathbb{R}^{2 n}$, the function $A$ is measurable. Integrating and changing variables as usual,

$$
\int_{B_{1}} A\left(a_{0}\right) d a_{0} \leq \sum_{k \in \mathbb{N}} \int_{B_{1}}\left\|f_{k}\right\|_{L^{1}\left(B_{2}\right)} d x^{\prime} \leq\left|B_{1}\right| \sum_{k \in \mathbb{N}}\left\|f_{k}\right\|_{L^{1}\left(B_{2}\right)}<\infty .
$$

Therefore $A\left(a_{0}\right)<\infty$ almost everywhere, which concludes the proof.

Lemma A.2 (Chain rule). Let $\psi \in W^{1, \infty}\left(B_{1} ; \bar{B}_{1}\right), u \in W^{1,1}\left(B_{2} ; \mathbb{R}^{m}\right)$. Then for almost all $a_{0} \in B_{1}$ the function $w(x)=u\left(a_{0}+\psi\left(x-a_{0}\right)\right)$ belongs to $W^{1,1}\left(B\left(a_{0}, 1\right) ; \mathbb{R}^{m}\right)$ with

$$
D w(x)=D u\left(a_{0}+\psi\left(x-a_{0}\right)\right) D \psi\left(x-a_{0}\right) .
$$

If $\psi(x)=x$ on $\partial B_{1}$ then $w=u$ (as traces) on $\partial B\left(a_{0}, 1\right)$.

Proof. We choose a sequence $u_{k} \in C^{\infty}\left(\overline{B_{2}} ; \mathbb{R}^{m}\right)$ such that $\left\|u_{k}-u\right\|_{W^{1,1}\left(B_{2}\right)} \leq$ $2^{-k}$ and apply Lemma A.1 to the sequence $f_{k}=\left(u_{k}-u, D u_{k}-D u\right) \in$ $L^{1}\left(B_{2} ; \mathbb{R}^{m} \times \mathbb{R}^{m \times n}\right)$, which obeys $\sum\left\|f_{k}\right\|_{L^{1}} \leq 2$. For any fixed $a_{0}$ not in the null set given by the lemma, we obtain the corresponding sequence $z_{k}$ with the properties asserted in Lemma A.1. Additionally we define $w_{k}$ by $w_{k}(x)=u_{k}\left(a_{0}+\psi\left(x-a_{0}\right)\right)$ and $w$ as in the statement. Each of the functions $z_{k}$ with values in $\mathbb{R}^{m} \times \mathbb{R}^{m \times n}$ is measurable, therefore the first $m$ components which are given by $w_{k}-w$ are measurable. The continuity of $w_{k}$ implies the measurability of $w$. Furthermore,

$$
\begin{aligned}
\left\|w_{k}-w\right\|_{L^{1}\left(B\left(a_{0}, 1\right)\right)} & =\int_{B\left(a_{0}, 1\right)}\left|u_{k}-u\right|\left(a_{0}+\psi\left(x-a_{0}\right)\right) d x \\
& \leq \int_{B\left(a_{0}, 1\right)}\left|f_{k}\right|\left(a_{0}+\psi\left(x-a_{0}\right)\right) d x=\left\|z_{k}\right\|_{L^{1}\left(B\left(a_{0}, 1\right)\right)} \rightarrow 0 .
\end{aligned}
$$

We conclude $w \in L^{1}$ and $w_{k} \rightarrow w$ in $L^{1}$.

We now repeat the procedure for the gradient. We denote by $F(x)=$ $D u\left(a_{0}+\psi\left(x-a_{0}\right)\right) D \psi\left(x-a_{0}\right)$ the expression given in the statement. Since every $u_{k}$ is smooth by the usual chain rule we obtain

$$
D w_{k}(x)=D u_{k}\left(a_{0}+\psi\left(x-a_{0}\right)\right) D \psi\left(x-a_{0}\right),
$$


which is the product of a continuous and an $L^{\infty}$ function and therefore measurable. Further,

$$
\left(D w_{k}-F\right)(x)=\left(D u_{k}-D u\right)\left(a_{0}+\psi\left(x-a_{0}\right)\right) D \psi\left(x-a_{0}\right) .
$$

The first factor is the second component of $z_{k}$ hence measurable by Lemma A.1, the second belongs to $L^{\infty}$. Continuity of $D w_{k}$ gives measurability of $F$. Further,

$$
\begin{aligned}
\left\|D w_{k}-F\right\|_{L^{1}\left(B\left(a_{0}, 1\right)\right)} & \leq\|D \psi\|_{\infty} \int_{B\left(a_{0}, 1\right)}\left|D u_{k}-D u\right|\left(a_{0}+\psi\left(x-a_{0}\right)\right) d x \\
& \leq\|D \psi\|_{\infty} \int_{B\left(a_{0}, 1\right)}\left|f_{k}\right|\left(a_{0}+\psi\left(x-a_{0}\right)\right) d x \rightarrow 0 .
\end{aligned}
$$

Therefore $F \in L^{1}$ and $D w_{k} \rightarrow F$ in $L^{1}$. Continuity of the distributional derivative implies $F=D w$ distributionally and $w \in W^{1,1}$.

To obtain the condition on the trace it suffices to extend $\psi$ to be the identity outside $B_{1}, u$ to a function in $W^{1,1}\left(\mathbb{R}^{n} ; \mathbb{R}^{m}\right)$ and work on a larger ball.

\section{B Construction for submultiplicative integrands}

We show here how our construction of the recovery sequence can be extended to the more general situation discussed in Remark 2.3. We focus on the orientation-preserving case, the other one is simpler. For brevity we only show how the basic construction step is modified, the covering of Lemma 3.3 is not significantly changed. Indeed, it suffices to use $p=1$ and takes Lebesgue points of $D u$ and $W(D u)$ instead of Lebesgue points of $D u$ and $\theta(\operatorname{det} D u)$; $W(D u) \in L^{1}$ by the growth condition (2.4).

Lemma B.1. Assume that $W \in C^{0}\left(\mathbb{R}_{+}^{n \times n},[0, \infty)\right)$ satisfies

$$
\frac{1}{c}|F|-c \leq W(G)
$$

and

$$
W(F G) \leq c_{W}(1+W(F))(1+W(G))
$$

for all $F, G \in \mathbb{R}_{+}^{n \times n}$, with a fixed $c_{W}>0$. Then for any $F \in \mathbb{R}_{+}^{n \times n}$ and $\eta>0$ there is $\delta>0$ such that for any $B=B\left(x_{0}, r\right)$ and $u \in W^{1,1}\left(B, \mathbb{R}^{n}\right)$ with

$$
f_{B}(|D u-F|+|W(D u)-W(F)|) d x \leq \delta \text { and } \operatorname{det} D u>0 \text { a.e. }
$$

there are $a_{0} \in B\left(x_{0}, r / 2\right)$ and $z \in W^{1,1}\left(B, \mathbb{R}^{n}\right)$ with $\operatorname{det} D z>0$ a.e., $z=u$ on $B\left(x_{0}, r\right) \backslash B\left(a_{0}, r / 2\right)$ and

$$
\int_{B\left(a_{0}, r / 2\right)} W(D z) d x \leq \int_{B\left(a_{0}, r / 2\right)}\left(W^{\mathrm{qc}}(D u)+\eta\right) d x .
$$


Additionally,

$$
\int_{B}|u-z| d x \leq \operatorname{cr} \int_{B}\left(W^{\mathrm{qc}}(D u)+1\right) d x .
$$

If $u$ is Lipschitz, then so is $z$.

Proof. This is very similar to the proof of Lemma 3.2, we only discuss the differences. After (3.3), (B.2) implies $W\left(F^{-1} D \varphi_{\eta}\right) \in L^{1}$ and equation (3.4) is replaced by

$$
\int_{B_{r / 2} \cap\left\{\operatorname{det} D \varphi_{\eta}<\gamma\right\}}\left(1+W\left(F^{-1} D \varphi_{\eta}\right)\right) d x \leq \frac{\left|B_{r / 2}\right|}{c_{W}(2+W(F))} \eta .
$$

In Lemma 3.1 we use $f=|D u-F|+|W(D u)-W(F)|$ and $g=1+$ $W\left(F^{-1} D \varphi_{\eta}\right),(3.5)$ is replaced by

$$
f_{B^{\prime}}\left(1+W\left(F^{-1} D \widehat{\varphi}_{\eta}\right)\right)(|D u-F|+|W(D u)-W(F)|) \circ v d x \leq c_{\eta} \delta,
$$

where $c_{\eta}=2^{n} f_{B_{r / 2}}\left(1+W\left(F^{-1} D \varphi_{\eta}\right)\right) d x<\infty$. In (3.7) we use continuity of $W$ instead of $\theta$. The remaining differences are in the treatment of the two error sets. We replace (3.9) by

$$
W(D z) \leq c_{W}(1+W(D u) \circ v)(1+W(D v)) .
$$

We start from $\omega$. From $|D u-F| \circ v \geq \varepsilon$ we deduce

$$
\begin{aligned}
1+W(D u) \circ v & \leq 1+W(F)+|W(D u)-W(F)| \circ v \\
& \leq c_{F, \varepsilon}(|D u-F|+|W(D u)-W(F)|) \circ v
\end{aligned}
$$

where $c_{F, \varepsilon}=1+(1+W(F)) / \varepsilon$. Therefore the estimate (B.5) gives

$$
\int_{\omega} W(D z) d x \leq c_{W} c_{F, \varepsilon} \int_{B^{\prime}}(1+W(D v))(|D u-F|+|W(D u)-W(F)|) \circ v d x
$$

Recalling (B.4), which had been obtained by the choice of $a_{0}$, we get

$$
\int_{\omega} W(D z) d x \leq c_{W} c_{F, \varepsilon} c_{\eta} \delta .
$$

The constant depends on $\varepsilon$ and $F$ (and hence on $\eta$ ) but not on $\delta$ and $u$.

In $\omega_{d}$ instead we have $|D u-F| \circ v \leq \varepsilon$. The continuity estimate (3.7) gives then $W(D u \circ v) \leq W(F)+1$ and therefore (B.5) reduces to

$$
W(D z) \leq c_{W}(2+W(F))(1+W(D v)) .
$$

With (B.3) we conclude

$$
\int_{\omega_{d}} W(D z) d x \leq\left|B^{\prime}\right| \eta .
$$

The conclusion is the same. 


\section{Acknowledgements}

This work was partially supported by the Deutsche Forschungsgemeinschaft through the Forschergruppe 797 "Analysis and computation of microstructure in finite plasticity", projects CO 304/4-2 (first author) and DO 633/2-2 (second author).

\section{References}

[1] Acerbi, E., And Fusco, N. Semicontinuity problems in the calculus of variations. Arch. Rat. Mech. Anal. 86 (1984), 125-145.

[2] Ball, J. M. Convexity conditions and existence theorems in nonlinear elasticity. Arch. Rational Mech. Anal. 63 (1976/77), 337-403.

[3] BALL, J. M. Global invertibility of Sobolev functions and the interpenetration of matter. Proc. Roy. Soc. Edinburgh Sect. A 88, 3-4 (1981), $315-328$.

[4] Ball, J. M. Discontinuous equilibrium solutions and cavitation in nonlinear elasticity. Philos. Trans. Roy. Soc. London Ser. A 306, 1496 (1982), 557-611.

[5] BALL, J. M. Some open problems in elasticity. In Geometry, mechanics, and dynamics. Springer, New York, 2002, pp. 3-59.

[6] Ball, J. M., And James, R. D. Fine phase mixtures as minimizers of the energy. Arch. Ration. Mech. Analysis 100 (1987), 13-52.

[7] Ball, J. M., And James, R. D. Proposed experimental tests of a theory of fine microstructure and the two-well problem. Phil. Trans. R. Soc. Lond. A 338 (1992), 389-450.

[8] Ball, J. M., ANd Murat, F. $W^{1, p}$-quasiconvexity and variational problems for multiple integrals. J. Funct. Anal. 58 (1984), 225-253.

[9] Bellido, J. C., And Mora-Corral, C. Approximation of Hölder continuous homeomorphisms by piecewise affine homeomorphisms. Houston J. Math. 37 (2011), 449-500.

[10] Ben Belgacem, H. Relaxation of singular functionals defined on Sobolev spaces. ESAIM Control Optim. Calc. Var. 5 (2000), 71-85 (electronic).

[11] Benešová, B., AND KRuŽík, M. Characterization of gradient Young measures generated by homeomorphisms in the plane. Preprint arXiv:1308.3377 (2013). 
[12] Bhattacharya, K. Microstructure of Martensite: Why it forms and how it gives rise to the Shape-Memory Effect. Oxford University Press, 2003.

[13] Conti, S. Quasiconvex functions incorporating volumetric constraints are rank-one convex. J. Math. Pures Appl. (9) 90, 1 (2008), 15-30.

[14] Conti, S., And DeLellis, C. Some remarks on the theory of elasticity for compressible neohookean materials. Ann. Scuola Norm. Sup. Pisa Cl. Sci. (5) (2003), 521-549.

[15] Conti, S., DeSimone, A., and Dolzmann, G. Soft elastic response of stretched sheets of nematic elastomers: a numerical study. J. Mech. Phys. Solids 50 (2002), 1431-1451.

[16] Conti, S., And Dolzmann, G. Relaxation of a model energy for the cubic to tetragonal phase transformation in two dimensions. Preprint arXiv:1403.4877 (2014).

[17] Conti, S., And Theil, F. Single-slip elastoplastic microstructures. Arch. Rat. Mech. Anal. 178 (2005), 125-148.

[18] Dacorogna, B. Direct methods in the calculus of variations, vol. 78 of Applied Mathematical Sciences. Springer-Verlag, Berlin, 1989.

[19] Daneri, S., And Pratelli, A. A planar bi-lipschitz extension theorem. Preprint arXiv:1110.6124 (2011).

[20] DeSimone, A., and Dolzmann, G. Macroscopic response of nematic elastomers via relaxation of a class of $\mathrm{SO}(3)$-invariant energies. Arch. Ration. Mech. Anal. 161 (2002), 181-204.

[21] Dolzmann, G., And DeSimone, A. Material instabilities in nematic elastomers. Physica D 136 (2000), 175-191.

[22] FonsecA, I. The lower quasiconvex envelope of the stored energy function for an elastic crystal. J. Math. pures et appl. 67 (1988), 175-195.

[23] Fonseca, I., Leoni, G., and Malý, J. Weak continuity and lower semicontinuity results for determinants. Arch. Ration. Mech. Anal. 178, 3 (2005), 411-448.

[24] Gol'dshtěrn, V. M., And Reshetnyak, Y. G. Quasiconformal mappings and Sobolev spaces, vol. 54 of Mathematics and its Applications (Soviet Series). Kluwer Academic Publishers Group, Dordrecht, 1990.

[25] Henao, D., and Mora-Corral, C. Invertibility and weak continuity of the determinant for the modelling of cavitation and fracture in nonlinear elasticity. Arch. Ration. Mech. Anal. 197, 2 (2010), 619-655. 
[26] Inaniec, T., Kovalev, L. V., and Onninen, J. Diffeomorphic approximation of Sobolev homeomorphisms. Arch. Ration. Mech. Anal. 201, 3 (2011), 1047-1067.

[27] Koumatos, K., Rindler, F., and Wiedemann, E. Differential inclusions and Young measures involving prescribed jacobians. Preprint arXiv:1312.1820 (2013).

[28] Koumatos, K., Rindler, F., And Wiedemann, E. Orientationpreserving Young measures. Preprint arXiv:130\%.1007 (2013).

[29] Kristensen, J. A necessary and sufficient condition for lower semicontinuity. Preprint (2014).

[30] Leoni, G., And Morini, M. Necessary and sufficient conditions for the chain rule in $W_{\text {loc }}^{1,1}\left(\mathbb{R}^{N} ; \mathbb{R}^{d}\right)$ and $\mathrm{BV}_{\mathrm{loc}}\left(\mathbb{R}^{N} ; \mathbb{R}^{d}\right)$. J. Eur. Math. Soc. (JEMS) 9, 2 (2007), 219-252.

[31] Mielke, A. Necessary and sufficient conditions for polyconvexity of isotropic functions. J. Convex Anal. 12, 2 (2005), 291-314.

[32] Morrey, Jr., C. B. Quasi-convexity and the lower semicontinuity of multiple integrals. Pacific J. Math. 2 (1952), 25-53.

[33] MüLLER, S. Higher integrability of determinants and weak convergence in $L^{1}$. J. Reine Angew. Math. 412 (1990), 20-34.

[34] Müller, S. Variational models for microstructure and phase transitions. In Calculus of variations and geometric evolution problems (1999), F. Bethuel et al., Eds., Springer Lecture Notes in Math. 1713, SpringerVerlag, pp. 85-210.

[35] Müller, S., Sivaloganathan, J., and Spector, S. J. An isoperimetric estimate and $W^{1, p}$-quasiconvexity in nonlinear elasticity. Calc. Var. Partial Differential Equations 8, 2 (1999), 159-176.

[36] Müller, S., AND Spector, S. J. An existence theory for nonlinear elasticity that allows for cavitation. Arch. Rational Mech. Anal. 131 (1995), 1-66.

[37] Müller, S., ANd Šverák, V. Convex integration with constraints and applications to phase transitions and partial differential equations. $J$. Eur. Math. Soc. (JEMS) 1 (1999), 393-442.

[38] Rindler, F. Talk at GAMM Annual Meeting (2014).

[39] RoubíčEK, T. Relaxation in optimization theory and variational calculus, vol. 4 of de Gruyter Series in Nonlinear Analysis and Applications. Walter de Gruyter \& Co., Berlin, 1997. 
[40] ŠIlhavÝ, M. Rank-1 convex hulls of isotropic functions in dimension 2 by 2. In Proceedings of Partial Differential Equations and Applications (Olomouc, 1999) (2001), vol. 126, pp. 521-529.

[41] ŠIlhavÝ, M. Ideally soft nematic elastomers. Netw. Heterog. Media 2 (2007), 279-311.

[42] Šverák, V. Regularity properties of deformations with finite energy. Arch. Rational Mech. Anal. 100 (1988), 105-127.

[43] Vodop'Janov, S. K., And Gol'DŠtě̃n, V. M. Quasiconformal mappings, and spaces of functions with first generalized derivatives. Sibirsk. Mat. Ž. 17, 3 (1976), 515-531, 715.

[44] Warner, M., And Terentuev, E. M. Liquid Crystal Elastomers. Oxford Univ. Press, 2003. 